Rapport - Société canadienne d'histoire de l'Église catholique

\title{
Monseigneur de Laval et les communautés de femmes
}

\section{Léo Hudon}

Volume 25, 1957-1958

URI : https://id.erudit.org/iderudit/1007442ar

DOI : https://doi.org/10.7202/1007442ar

Aller au sommaire du numéro

Éditeur(s)

La Société canadienne d'histoire de l'Église catholique

ISSN

0318-6148 (imprimé)

1927-7075 (numérique)

Découvrir la revue

Citer cet article

Hudon, L. (1957). Monseigneur de Laval et les communautés de femmes. Rapport - Société canadienne d'histoire de l'Église catholique, 25, 35-57.

https://doi.org/10.7202/1007442ar

Tous droits réservés @ La Société canadienne d'histoire de l'Église catholique, 1958
Ce document est protégé par la loi sur le droit d'auteur. L'utilisation des services d'Érudit (y compris la reproduction) est assujettie à sa politique d'utilisation que vous pouvez consulter en ligne.

https://apropos.erudit.org/fr/usagers/politique-dutilisation/ 


\section{Monseigneur de Laval et les communautés de femmes}

\section{Introduction}

Un conférencier, lors de la présentation d'un travail, aux fêtes du Deuxième Centenaire de $M^{\mathrm{gr}}$ de Laval, se disait soumis au supplice du lit de Procuste. Ma situation ne serait-elle pas un peu la même? Le sujet au programme renferme quatre études, dont chacune demanderait plus d'une heure.

Dans la présente causerie, il en serait comme lors de la visite d'un musée ou d'une exposition: les différents points esquissés ou étudiés pourraient ressembler à des gouaches ou à des fusains offrant de simples évocations; à des fresques présentant un fait historique, la synthèse d'une cuvre, les grandes lignes d'un projet; à des peintures fixant les traits d'une physionomie.

La venue en Nouvelle-France d'un Vicaire apostolique posait un problème. On était surpris de voir que le Prélat ne fut pas évêque titulaire. " $\mathrm{M}^{\mathrm{gr}}$ notre Prélat est ici, non pas sous le titre $\mathrm{d}^{\prime} E v e ̂ q u e ~ d e$ Québec ou du Canada, écrit la Mère Juchereau de Saint-Ignace, mais comme Commissaire apostolique, sous le titre étranger d'Evêque de Pétrée; et ce titre a fait parler bien du monde.»

Aussi, « à peine $\mathrm{M}^{\mathrm{gr}}$ de Laval fut-il débarqué, continue la Mère Juchereau de Saint-Ignace, qu'il y eût plusieurs discussions pour savoir à qui les Communautés obéiraient, et nous nous trouvâmes assez embarrassées. M. de Queylus avait les pouvoirs de $M^{\text {gr }}$ l'ÁArchevêque de Rouen qui jusqu'alors avait été reconnu pour le Supérieur du pays, et bien des personnes disaient que cet Archevêque était au-dessus de $\mathbf{M}^{\mathrm{gr}}$ de Laval, qui n'était que Vicaire apostolique. Mais, après avoir bien consulté Dieu, et demandé les sentiments de personnes plus éclairées, nous nous soumîmes à $\mathbf{M}^{\text {gr }}$ de Laval.»

Il n'y avait pas d'autre parti à prendre. Une lettre de cachet de la Reine, en date du 31 mars 1659, ordonne même de faire repasser en France tous ceux qui refuseraient de se soumettre à l'autorité du Vicaire apostolique. La Reine déclarait que telle était aussi l'intention du Roi, son fils. $\mathbf{M}^{\mathrm{gr}}$ de Laval est donc reconnu seul Supérieur des deux Communautés; et pour écarter tout doute à ce sujet, il prend le titre de Supérieur dans les actes relatifs à ces maisons.

\section{I. - LES HOSPITALIÈRES DE QUÉBEC.}

La première partie de notre travail est consacrée aux Hospitalières de Québec: un simple rappel des séjours de $\mathbf{M}^{\mathrm{gr}}$ de Laval à l'Hôtel-Dieu, de la visite canonique de 1660 , du projet d'établissement des Hospitalières 
de Québec à Montréal, et un hommage à Mère Catherine de SaintAugustin.

\section{1) Les séjours}

En arrivant à Québec, après avoir passé quelques jours chez les Pères Jésuites, $\mathbf{M}^{\mathrm{gr}}$ se rend à l'Hôtel-Dieu où il occupe un appartement dépendant de l'Hôpital, il y demeure près de trois mois avec les prêtres de sa suite.

Chez les Hospitalières, dit Latour, l'Evêque est traité autant que la pauvreté de la maison le permet, avec beaucoup de propreté et de zèle quoique très simplement. Mais cette simplicité ne lui suffit pas; il se plaint toujours qu'on en fait trop, affecte une sorte d'avidité pour tout ce qu'il y a de moins bon.

Dans son amour de la pauvreté, dira l'abbé Joseph de la Colombière, $M^{\mathrm{gr}}$ de Laval retournera plus tard à l'Hôtel-Dieu avec l'intention de s'y fixer pour toujours. On n'ose s'opposer à cette résolution, de peur de faire trop de violence à son attrait et à sa ferveur. Il a fallu du temps et employer les négociations de quelques personnes de piété pour le faire revenir au Séminaire.

\section{2) La visite canonique de 1660}

Après la visite pastorale sur le côte de Beaupré, au début de 1660 , $\mathrm{M}^{\mathrm{gr}}$ de Laval entreprend celle des Communautés et commence par l'HôtelDieu. Ce Monastère compte alors quinze Religieuses, dont la Mère Catherine de Saint-Augustin. Il ne peut s'empêcher d'admirer leurs vertus, leur dévouement pour les malades, leur renoncement à toutes les choses terrestres. Il se croit même obligé d'adoucir un peu l'austérité de leurs règles et de retrancher certains jeûnes qu'il pensait incompatibles avec leurs travaux. Il renvoie aussi les pensionnaires qu'elles avaient gardées jusque-là, afin qu'elles puissent se livrer exclusivement à l'œuvre de leur Institut. Il approuve les professions faites depuis l'établissement du Monastère en Nouvelle-France, pour huit Religieuses; nomme M. Charles de Lauzon-Charny, supérieur du Monastère.

\section{3) Le projet d'établissement à Montréal}

Le point historique le plus saillant dans l'histoire de l'Hôtel-Dieu de Québec pour cette époque, est le projet d'établissement des Hospitalières de Québec à Montréal. Qui serait l'auteur ou l'initiateur de ce projet? Quels étaient les arguments en faveur de cet établissement ?

L'annaliste de l'Hôtel-Dieu note que le 28 août 1659, fête de saint Augustin, $\mathrm{M}^{\mathrm{gr}}$ de Laval officie pontificalement à l'Hôpital; elle ajoute qu'il ne tarda guère à faire voir combien il s'intéressait à leurs affaires; et qu'il entrait dans le dessein qu'avait eu M. de Queylus de les établir à Montréal, jugeant qu'en effet ce serait un bien de n'avoir en Canada qu'un seul Institut d'Hospitalières.

M. de Queylus, supérieur des Prêtres de Saint-Sulpice, installé définitivement à Ville-Marie, pensait qu'il n'était pas nécessaire, ni même sage de faire venir de France une nouvelle Communauté d'Hospitalières 
pour prendre charge de l'Hôtel-Dieu de Ville-Marie; il savait les autres pour l'instant sans ressources, incapables de subsister et sans aide, dans une Colonie où tout coûtait très cher; de plus cette Communauté n'avait pas encore été érigée en Ordre religieux.

Durant l'hiver qu'il venait de passer à Québec en sa qualité de Grand Vicaire de l'Archevêque de Rouen, les devoirs de son ministère l'avaient souvent mis en relation avec les Religieuses de l'Hôtel-Dieu. Il en avait apprécié l'esprit religieux, les services, le dévouement, le savoir-faire. Il avait décidé de les appeler dans un avenir prochain à Ville-Marie. La Mère Juchereau de Saint-Ignace, dans ses Mémoires, a inséré le souvenir des attentions bienveillantes de M. de Queylus à leur égard, qui jusqu'à la fin de sa vie donne à l'Hôtel-Dieu de Québec des preuves de sa bien vive considération.

Il suffit de rappeler l'acte signé le $1^{\text {er }}$ mars 1675 , en présence de $\mathrm{M}^{\mathrm{gr}}$ de Laval, à Clermont, France. Il s'agit de la fondation de 6,000 livres, dont 1,000 livres pour les pauvres, et 5,000 livres pour la dot d'une religieuse de chœur. "M. de Queylus déclare qu'il a été profondément édifié de la grande piété, charité et autres bonnes œuvres des Religieuses Hospitalières de Québec en la Nouvelle-France, et qu'il désire participer à leurs mérites.»

M. de Queylus, en sa qualité de membre de la Société de NotreDame de Montréal, ne pouvait sans doute ignorer tout à fait le désir de ses co-associés. Mais cela n'avait guère fait d'impression sur son esprit, n'ayant été admis en la Compagnie qu'au moment de son départ pour le Canada. A la vérité, il demeurait un peu étranger à tous les vœux de la première heure. Savait-il bien, continue Mlle Daveluy, que M. de la Dauversière n'avait fondé justement cette Communauté d'Hospitalières de La Flèche qu'en vue de son installation, un jour, dans le lointain Montréal ?

Comment M. de Queylus s'y prendra-t-il pour amener les Hospitalières de Québec à Montréal ? Mlle Mance projette un voyage en France dans l'intérêt de l'Hôpital de Ville-Marie, elle soumet au Supérieur des Prêtres de Saint-Sulpice ses motifs. M. de Queylus ne veut ni peiner ni blesser Mlle Mance toujours souffrante en lui annonçant, séance tenante, sans ménagement, des projets qu'il savait si bien venir à l'encontre des siens, il préfère garder le silence et user de stratagème.

Quelques jours après cet entretien, M. Gabriel Souart, prêtre de Saint-Sulpice, en compagnie du Père Simon LeMoyne, jésuite, se rend à Québec; le Sulpicien frappe à la porte de l'Hôtel-Dieu et demande à voir la Mère Marie-Renée Bouillé de la Nativité, que son supérieur M. de Queylus lui avait dit fort souffrante et avoir besoin, pour se rétablir, d'un changement d'air complet. D'après Faillon, M. Souart ne connaissant pas les intentions de M. de Queylus, croit qu'il s'agit simplement de faire une visite professionnelle auprès de cette Religieuse et lui procurer quelques soulagements par ce changement de lieu.

M. Souart revenait à Ville-Marie à la fin de septembre en compagnie de Mère Marie-Renée Bouillé de la Nativité, de Mère Jeanne-Thomas 
Agnès de Saint-Paul, sa compagne, et du Père Pierre-Marie Chaumonot, jésuite. Le plus difficile reste à faire: avertir Mlle Mance de l'arrivée bien étonnante des deux Religieuses de Québec.

M. de Queylus demande à parler à Mlle Mance: "Voilà deux bonnes filles hospitalières qui arrivent, lui dit-il, à cause que l'une d'elles a eu besoin de changer d'air, elles vont venir vous saluer et vous demander le couvert.» C'était vraiment, ajoute Mlle Daveluy, procéder de façon qui donnait à réfléchir à un esprit perspicace. Mlle Mance paraît un instant interdite, mais se ressaisit vite et accueille avec sa grâce ordinaire les deux Religieuses. "Vous venez, mes Mères, s'exclama-t-elle avec un sourire, et moi je m'en vais.»

Mlle Mance se rendra auprès de $\mathrm{M}$. de Maisonneuve qui la reçoit de façon un peu glaciale. Le Gouverneur venait d'apprendre par un tiers, l'arrivée de deux Hospitalières à l'Hôpital de Ville-Marie, avec le dessein d'y demeurer tout l'hiver en l'absence de l'administratrice. Cela donnait à penser que Mlle Mance n'avait pas dû être tout à fait étrangère à une aussi grave détermination; les explications remirent tout au point. S'apercevant tous deux, remarque Dollier de Casson "qu'ils n'étaient pas plus savants l'un que l'autre en cette matière et que ces bonnes filles ne venaient que pour prendre l'air, afin de se guérir, ils se mirent à rire de la fausse alarme et se séparèrent bons amis ».

Il restait tout de même quelques mesures à prendre afin de sauvegarder les intérêts de chacun. Mille Mance, en sa qualité d'administratrice de l'Hôpital, nomme Madame de la Bardillière pour la remplacer officiellement, et lui recommande de ne rien abandonner de la conduite de l'Hôpital à qui que ce soit, pour quelque raison que ce fut, durant son absence; puis s'étant assurée que les Religieuses auraient tout le confort et les attentions possibles, elle annonce son départ de Ville-Marie pour les derniers jours de septembre. La Mère Bourgeois passant aussi en France pour y chercher des coopératrices, avait prié les deux Hospitalières de Québec de faire la classe aux enfants pendant son absence; elles acceptèrent bien volontiers ce charitable emploi, sagement conseillées par M. de Queylus.

Avant son départ pour la France, Mlle Mance passe huit jours à Québec chez les Religieuses de l'Hôtel-Dieu, enchantées de la recevoir et de la remercier ainsi de la cordiale réception faite à leurs Sours actuellement à Ville-Marie. Pendant ce séjour, elle promet aux Religieuses de faire, selon leur désir, des démarches auprès de Madame la duchesse d'Aiguillon, pour une fondation en faveur d'un nouvel établissement de la Communauté de Québec à Ville-Marie, qu'elles essayeraient ensuite de faire accepter aux Associés de Notre-Dame de Montréal.

Un an plus tard, nos deux Hospitalières seront invitées par $\mathrm{M}^{\mathrm{gr}}$ de Laval à retourner à leur Communauté; celles de La Flèche avaient obtenu le 2 octobre 1659, le document les autorisant à se rendre à Ville-Marie.

Voyons-les maintenant sur le chemin du retour. Celles venant de LaFlèche, après un arrêt de quelques semaines à Québec, partent pour Montréal. Les vents contraires ralentissent leur navigation de telle sorte 
qu'elles doivent demeurer sur le fleuve plusieurs jours. A peu de distance de l'Ile de Montréal, l'on aperçoit une barque venant en sens contraire, mais à une grande vitesse ayant le vent en poupe. Lorsqu'on en est à dix ou quinze pas, distance qu'on ne peut rendre meilleure à cause de la température, chacun se reconnaît, et de brèves salutations s'échangent. La barque qui descend contient les deux Hospitalières de Québec. Elles retournent dans leur maison en compagnie de M. Souart. La Providence en avait décidé.

Le projet d'établissement des Hospitalières a la vie dure. M. de Queylus n'abandonne pas son idée. Il conçoit alors le dessein de fonder à Ville-Marie un hospice, pour y recevoir et traiter gratuitement les sauvages invalides, aussi ceux parvenus à un grand âge, qui voudraient s'y retirer.

Il offre aux Hospitalières de Québec la conduite de cet hospice projeté, voulant ainsi remplir ses promesses envers elles, sans nuire aux Hospitalières de LaFlèche, et $\mathbf{M}^{\mathrm{gr}}$ de Laval, à qui $M$. de Queylus avait soumis son dessein, l'approuve de grand cœur.

Le 11 novembre 1671, l'intendant Talon écrivant au ministre Colbert au sujet de cet hospice, avoue ne le faire qu'après en avoir été prié par M. l'Evêque de Pétrée, par M. de Queylus, et par la Supérieure des Hospitalières de Québec; cette dernière s'obligeait à donner des Religieuses pour la conduite de cet établissement. On ne demande que l'agrément du Roi et des Lettres. Le ministre Colbert répond l'année suivante à l'intendant Talon, que le Roi espérait que M. de Queylus demeurerait ferme dans le dessein d'établir et de fonder cet hôpital.

\section{4) Mère Catherine de Saint-Augustin}

L'Hôtel-Dieu de Québec comptait alors parmi ses religieuses plusieurs âmes de choix. La Servante de Dieu, Mère Catherine de Saint-Augustin, attire notre attention. Elle fut pour l'Hôtel-Dieu de Québec ce que Mère Marie de l'Incarnation était pour les Ursulines; un trésor de grâces, de vertus et de mérites. Les voies par lesquelles la Providence conduisit ces deux saintes personnes furent bien différentes.

$\mathrm{M}^{\mathrm{gr}}$ de Laval, le 24, août 1659, avait dû lui conférer le sacrement de Confirmation, les circonstances n'ayant pas permis qu'elle le reçut avant de quitter la France. Il connaissait le trésor de sainteté que possédait l'Hôtel-Dieu; pendant les trois mois de son séjour à l'Hôpital, après son arrivée, il dut la voir à l'œuvre, examiner de près sa conduite et juger par lui-même de l'esprit qui la guidait. On peut croire que le Père Paul Ragueneau n'avait pas été lent à la faire connaître à l'Evêque. Chose certaine, il tint toujours cette Religieuse en très haute estime, à cause de sa prudence, de sa sainteté et des vertus surnaturelles.

$\mathbf{M}^{\mathrm{gr}}$ de Laval avait une mission spéciale à remplir au Canada, celle de fonder et d'organiser sur les bords du Saint-Laurent une Eglise destinée à être la mère des Eglises de ce continent. La Mère Catherine de Saint-Augustin fait sienne l'œuvre du premier évêque de Québec, qui trouvait en elle peut-être le plus puissant de ses auxiliaires. On l'a justement appelée la Catherine de Sienne du Canada. 
Représentons-nous l'Evêque de Québec se rendant au parloir des Hospitalières pour se recommander aux prières ferventes de la Servante de Dieu, la consulter et l'intéresser au succès des affaires les plus importantes de son Vicariat. Il ordonnera, peu de temps après la mort de Mère Catherine de Saint-Augustin, que l'on recueille les lettres, les notes spirituelles, tous les documents capables d'éclairer sa vie pour les envoyer au Père Paul Ragueneau, jésuite, qui doit préparer une biographie.

$M^{\mathrm{gr}}$ de Laval écrit lui-même à la Supérieure des Hospitalières de Bayeux: "Ma très chère Mère, il $\mathrm{y}$ a grand sujet de bénir Dieu de la conduite qu'il a tenue sur notre Sœur Catherine de Saint-Augustin. C'était une âme qu'il s'était choisie pour lui communiquer des grâces très grandes et très particulières. Sa sainteté sera mieux connue dans le Ciel qu'en cette vie, car assurément elle est très extraordinaire.

"Elle a beaucoup souffert avec une fidélité inviolable et un courage qui était au-dessus du commun. Sa charité pour le prochain était capable de tout embrasser pour difficile qu'il fût. Je n'ai pas besoin des choses extraordinaires qui se sont passées en elle pour être convaincu de sa sainteté; ses véritables vertus me la font parfaitement connaître. Dieu a fait une faveur bien particulière à nos Hospitalières de Québec et même à tout le Canada, lorsqu'il y a envoyé cette âme qui lui était si chère.»

Terminons par un jugement de Mère Marie de l'Incarnation: "J'ai entendu dire de $\mathrm{M}^{\mathrm{gr}}$ notre Prélat que cette bonne Mère était l'âme la plus sainte qu'il eût connue. Il en pouvait parler avec connaissance, car c'est lui qui la dirigeait.»

\section{II. - MONSEIGNEUR DE LAVAL ET LES URSULINES.}

Rendons-nous à la rue du Parloir, chez les Religieuses Ursulines; rappelons le séjour de $\mathbf{M}^{\mathrm{gr}}$ de Laval, les visites canoniques de 1660 et de 1681, le projet d'établissement à Montréal, et saluons la mémoire de Mère Marie de l'Incarnation.

\section{1) Le séjour}

Pour ne pas enlever aux malades des pièces de l'hôpital, $\mathrm{M}^{\mathrm{gr}} \mathrm{de}$ Laval décide d'aller loger chez les Ursulines. Mère Marie de l'Incarnation écrit en novembre 1659: "Nous lui avons prêté notre séminaire qui est à un des coins de notre clôture et tout proche de la paroisse. $\mathrm{M}^{\mathrm{gr}}$ aura la commodité et l'agrément d'un beau jardin, et afin que nous soyons logées selon les saints canons, il a fait faire une clôture de séparation. Nous en serons incommodées, parce qu'il nous faut loger nos séminaristes dans nos appartements, mais le sujet le mérite, et nous porterons avec plaisir cette incommodité jusqu'à ce que la maison épiscopale soit bâtie.»

Le Récit ajoute: "nous fûmes obligés d'abandonner la salle de communauté des Religieuses pour servir de classe. $M^{\text {gr }}$ demeura chez nous environ deux ans. La tradition désigne encore l'endroit où le Prélat célébrait tous les jours la sainte messe.» 
$\mathrm{M}^{\mathrm{gr}} \mathrm{y}$ demeure jusqu'au 6 novembre 166l. Il nous apprend lui-même avoir loué la petite maison de Madame de la Peltrie, au coin des rues Des Jardins et Donacona, pour deux cents livres par année. "Nous la trouvâmes assez riche, disait-il, parce qu'elle suffit à notre pauvreté. Nous avons trois prêtres, qui sont nos commensaux, et deux serviteurs."

Les Mères Ursulines devaient être heureuses d'héberger l'Evêque de Québec, si l'on en juge par leurs sentiments à son égard: "... c'est un bonheur, écrit Mère Marie de l'Incarnation, d'avoir un homme dont les qualités personnelles sont si rares. Sans parler de sa naissance qui est fort illustre, c'est un homme de mérite et d'une vertu singulière... Ce ne sont par les hommes qui l'ont choisi... Je dis avec vérité qu'il vit saintement et en apôtre, qu'il ne sait ce que c'est que le respect humain. Je vous ai dit qu'on n'attendait pas d'Evêque cette année. Aussi n'a-t-il rien trouvé de prêt pour le recevoir quand il est arrivé.»

"C'est bien l'homme du monde, dira-t-elle encore, le plus austère et le plus détaché des biens d'ici-bas; il donne tout et vit en pauvre; et l'on peut dire, avec vérité, qu'il a l'esprit de pauvreté. Il pratique cette pauvreté en sa maison, en son vivre, en ses meubles, en ses domestiques; car il n'a qu'un jardinier, qu'il prête aux pauvres gens quand ils en ont besoin, et un homme de chambre."

\section{2) La visite canonique de 1660}

Au mois d'avril $1660, \mathrm{M}^{\mathrm{gr}}$ de Laval fait sa première visite canonique du Monastère que dirige Mère Marie de l'Incarnation. Les Religieuses sont grandement consolées de voir que le zélé Prélat s'occupe si tôt de leurs intérêts personnels.

Il y trouve la même ferveur qu'à l'Hôtel-Dieu, un dévouement sans borne pour l'éducation des jeunes filles et surtout des jeunes filles sauvages. La vue de ces pauvres enfants des bois, que les Religieuses essayent de former à la piété, à la vertu, à l'amour du travail, enflamme son cœur d'un saint zèle. C'est alors qu'il fait entrer vingt pensionnaires. C'est un mystère, que ce saint Evêque, avec si peu de ressources à sa disposition, ait pu suffire à tant de bonnes euvres.

Un incident, lors de cette visite, donne toutefois de l'inquiétude aux Mères. Quelques Religieuses demandèrent à l'Evêque de leur donner " un abrégé de leurs Constitutions ", préparées en 1646 par le Père Jérôme Lalemant, jésuite, en conformité des exigences, des besoins et de la situation du pays... "Ces Constitutions avaient été dressées avec un soin admirable, et avec tant de déférence à nos sentiments, dit la Mère Marie de l'Incarnation, qu'il n'y a chapitre que chaque søur n'ait lu trois fois et dont elle n'ait conféré avec le Révérend Père, disant en toute liberté ses pensées et ses sentiments. Le chapitre était ensuite présenté à la Communauté pour être reçu par suffrages secrets; il n'y en a pas un seul que toutes nos Religieuses n'aient reçu. Il faut avouer qu'il ne se peut rien voir de mieux concerté ni de plus propre à notre dessein et Institut en ce pays.»

Monseigneur propose cependant d'y introduire quelques réformes qu'il juge utiles, entr'autres changements: que la maîtresse des novices 
le fut aussi des jeunes professes et que cette charge fut sujette à l'élection; il aurait voulu que l'on fit une réforme dans la manière de chanter les offices et que pour leur donner une teinte plus religieuse et plus sainte, on adoptât le chant à voix droite tel qu'il se pratique à la Visitation et au Carmel.

On comprend que ces modifications demandées, soulevèrent dans la Communauté certaines récriminations. Mère Marie de l'Incarnation, qui savait avec quelle bénédiction, on avait jusque-là observé ces Constitutions, en fut alarmée, "car, disait-elle, quand on est bien, il faut s'y tenir, parce qu'on est bien; mais en changeant, on ne sait si on sera bien ou mal ».

La vénérable Supérieure avoue que, sur leurs remontrances, Monseigneur n'ordonne le changement concernant l'élection de la maîtresse des novices, que pour trois ans et à titre d'essai. Quant au nouvel " abrégé des Constitutions ", l'Evêque leur donne toute une année pour réfléchir... "l'affaire est déjà toute pensée, écrit-elle, et la résolution toute prise; nous n'acceptons pas, si ce n'est à l'extrêmité de l'obéissance ". Puis elle ajouta: "Nous avons affaire à un Prélat d'une très haute sainteté, mais s'il est une fois persuadé qu'il y va de la gloire de Dieu, il n'en reviendra jamais, et il nous en faudra passer par là, ce qui causerait un grand préjudice à nos observances.»

$\mathrm{Au}$ bout de ce temps, voyant que les Religieuses persistaient dans « leurs sentiments ", l'Evêque laisse de côté le sien et confirme les Constitutions primitives, qui furent observées jusqu'à l'adoption des Constitutions de Paris, en 1681.

\section{3) La visite canonique de 1681}

Nouvelle tentative lors de la visite canonique de $\mathbf{M}^{\mathrm{gr}}$ de Laval en mars 1681. Plutôt que de mutiler les Constitutions préparées avec la collaboration du Père Jérôme Lalemant, il fut résolu par les autorités ecclésiastiques, d'accord avec celles du Monastère, que l'on adopterait à Québec les Constitutions du grand Couvent de Paris auquel le Monastère de Québec s'affilie.

Voici ce qu'en dit le Récit: "Au commencement de mars 1681, $\mathbf{M}^{\text {gr }}$ de Laval commence sa visite en notre maison et, après avoir parlé à toutes les Religieuses, il en fait la conclusion le 17 du même mois, ordonnant à la Communauté de laisser les Constitutions particulières que l'on avait composées pour cette maison, après son établissement, pour prendre les Constitutions et les règlements de la Congrégation de Paris, nous unissant à la dite Congrégation. Nous avons commencé le 20 mars à suivre l'ordre du jour tel qu'il est marqué dans les Constitutions; le 25 du même mois, nous prîmes la coiffure et la ceinture de cuir; pour le reste de l'habit, les cottes et les manteaux, ne se trouvant pas d'étoffe convenable, il faut attendre qu'il en vienne de France, et l'on en demande cette année."

\section{4) Le projet d'établissement à Montréal}

Le projet d'établissement des Religieuses Ursulines à Montréal est de beaucoup semblable à celui des Hospitalières de Québec. L'initiateur 
du projet serait le même, $\mathrm{M}^{\mathrm{gr}}$ l'Evêque y donne son approbation, cependant l'issue n'en est pas plus heureuse; seule Madame de la Peltrie fera un voyage! Voici des faits pouvant servir de jalons:

Madame de la Peltrie, si zélée pour le salut des âmes, se rend à Ville-Marie et fait tous les efforts imaginables, nous dit Faillon, pour attirer les Ursulines à Ville-Marie. S'étant déjà engagée verbalement à être leur fondatrice à Québec, où elle ne pouvait les aider qu'assez faiblement, son directeur lui ordonne de renoncer au dessein d'entreprendre une nouvelle fondation à Ville-Marie, au détriment de la première. Elle retourne donc à Québec et a besoin de toute sa vertu pour obéir. On l'aurait entendu assurer sur la fin de ses jours, que rien ne lui avait été plus sensible; mais enfin il fallait se restreindre au Monastère de Québec.

Une lettre du 24, septembre 1654, de Mère Marie de l'Incarnation, nous apprend: "L'on nous propose et l'on nous presse de nous établir à Montréal; mais nous n'y pouvons entendre si nous n'y voyons une fondation car on ne trouve rien de fait en ce pays, et l'on y peut rien faire qu'avec des frais immenses."

La demande de leur établissement à Montréal pouvait venir des familles de Ville-Marie. Il suffit de consulter les listes des anciennes élèves pour avoir le nombre de celles qui étaient obligées de se rendre à Québec, en canot l'été ou sur la glace en hiver; les grandes difficultés de déplacement faisaient souhaiter et espérer un Couvent des Ursulines à Ville-Marie.

Après l'arrivée de la Sœur Bourgeoys, avec ses compagnes à VilleMarie en 1659, on fait auprès des Ursulines de nouvelles instances. " On nous presse de nous établir à Montréal, écrivait de nouveau Mère Marie de l'Incarnation; mais nous ne sommes pas en état de le faire.» Le même obstacle qui les empêche de s'établir à Ville-Marie, lorsque la Seur Bourgeoys y arrive la première fois, le manque de ressources pécuniaires, ne permet pas non plus d'exécuter ces desseins en 1659.

Lorsque M. de Queylus arrive à Québec en 1668, les Mères s'empressent de lui donner des marques de leur estime, ainsi qu'aux Prêtres de Saint-Sulpice qu'il conduisait à Ville-Marie; et comme ces Religieuses ne perdaient pas l'espérance qu'elles avaient d'aller s'établir dans cette dernière ville, on fait alors quelques démarches pour engager M. de Queylus à favoriser lui-même leur dessein. Sans s'y refuser expressément, il répond d'abord que l'état des choses ne permettait pas encore de l'exécuter.

C'est apparemment ce qui fait dire à Mère Marie de l'Incarnation, dans une lettre de l'année 1670: «M. de Queylus, supérieur spirituel et temporel pour Messieurs de Saint-Sulpice, nous promet sa protection, lorsque les choses seront en état. Nous ne sommes pas marries de ce retardement, et $\mathbf{M}^{\mathrm{gr}}$ notre Prélat, qui ne fait rien qu'avec prudence, est aussi de ce sentiment.»

A l'occasion de l'établissement des Jésuites et des Récollets à VilleMarie, vers 1693 , on presse beaucoup les Ursulines d'exécuter enfin 
le projet formé autrefois, d'aller s'y fixer elles-mêmes. Mère Marie de Saint-Joseph, leur supérieure, écrivait en ces termes à M. Dollier de Casson: "Notre-Seigneur donne vocation à quelques religieuses de cette maison pour aller en vos quartiers, elles me prient comme je le fais ici avec plaisir, de vous demander votre protection et votre permission, sans lesquelles nous ne voulons pas penser à la chose. Je vous les demande donc très instamment, monsieur,... vous pouvez tout à Ville-Marie, et même à Québec, sur l'esprit de votre illustre prélat. Je pense monsieur, que vous n'ignorez pas que dans les commencements de Ville-Marie, nous y fûmes demandées, qu'on nous marqua même la place de l'établissement, et que Madame de la Peltrie, notre fondatrice, y monta et demeura deux ans dans ce dessein, avec une jeune fille qui depuis a été religieuse, et c'est une de nos anciennes. Vous feriez, monsieur, bien de la grâce à toute notre communauté, et à moi, de nous dire vos sentiments sur cette affaire; et si vous en seriez content, au cas que Dieu nous fît trouver les moyens de nous établir à Ville-Marie, car autrement nous ne voulons pas y penser. Nous connaîtrons la volonté de Dieu par votre réponse.»

M. Dollier de Casson ne put faire une réponse telle que la souhaitaient peut-être les Religieuses Ursulines. Il avait déjà écrit à $M^{\mathrm{gr}}$ de Saint-Vallier pour lui exposer les inconvénients qu'il voyait à l'exécution de ce projet; et le Prélat avait paru être touché de la solidité de ces raisons. "Si les Ursulines vous écrivent, avait-il répondu, je vous conseille de leur faire réponse, et de leur marquer à peu près les mêmes choses que vous m'exposez dans votre lettre, toutes les raisons que vous me donnez me paraissent bonnes et me font suspendre mon jugement.»

Les Mères persévérant toujours dans leur projet de s'établir à Montréal, prennent le parti de s'adresser directement à M. de Tronson, persuadées que tous les obstacles cesseraient s'il approuve lui-même leur dessein; leur Supérieure écrit donc et lui détaille tous les motifs qu'elle juge les plus propres à faire impression sur son esprit, l'assurant surtout que ce nouvel établissement ne nuirait en rien à celui de la Sœur Bourgeoys.

M. de Tronson, qui n'aurait pas entendu parler de ce projet, lui répond en ces termes: "J'ai vu, par votre lettre du mois d'octobre dernier, la crainte où vous êtes que la pensée de vous établir présentement à Ville-Marie ne m'ait fait de la peine. Mais comment m'en aurait-elle fait, puisque je ne sais rien de cet établissement que ce que vous m'en écrivez? Je n'ai entendu dire qu'à vous, qu'on vous y souhaitait, qu'on vous y donnait une maison avec un jardin, que votre établissement ne nuirait point aux filles de la Congrégation, et qu'il y aurait de quoi travailler pour les unes et pour les autres.

"Pour vous parler avec sincérité, je vous dirai que des personnes qui connaissent le pays, ont peine à croire que deux Communautés de filles qui ont les mêmes emplois, ne soient point de trop pour Ville-Marie. Tout ce que je puis faire, c'est d'écrire cette année à nos messieurs pour être éclairé là-dessus; et la suivante, après que par leur réponse, ils nous auront mandé tout le détail de cette affaire, nous vous ferons savoir 
exactement ce que nous en pensons. Il ne s'agit que de bien connaître la volonté de Dieu. De peur que, comme il arrive quelquefois, en voulant trop multiplier le bien et le trop étendre, on ne l'affaiblisse et on ne le diminue... J'espère que vos prières contribueront à la faire connaître.»

(signé) Tronson.

La réserve de $M$. de Tronson, en répondant de la sorte, dut faire comprendre aux Religieuses qu'au jugement de cet homme sage et prudent, leur dessein n'était pas dans l'ordre de la Providence. Ainsi nous ne voyons pas que depuis elles aient jamais renouvelé leur demande, ne désirant de leur côté connaître à cet égard que la volonté de Dieu.

Quoiqu'il en soit, les Messieurs du Séminaire, chargés seuls comme seigneurs de soutenir la Colonie et de pourvoir à ses divers besoins, ne goûtaient pas le projet d'établir les Religieuses Ursulines à Ville-Marie; ils pensaient que ces Religieuses, vivant en clôture, étaient moins en état que les Sœurs de la Congrégation, de rendre au pays le genre de services qu'exigeaient alors ses besoins. Ils les remercièrent d'ailleurs en alléguant ce motif. Ces Religieuses qui ne pouvaient encore vivre de leur revenu ni se suffirent à elles-mêmes par le travail comme faisaient les Sœurs de la Congrégation, auraient été à charge au pays. Leur établissement eut été même inutile, la Colonie de Montréal était trop peu nombreuse pour fournir assez d'occupation à deux Communautés à la fois.

\section{5) Mère Marie de l'Incarnation}

Terminons notre visite chez les Ursulines. Saluons la mémoire de Mère Marie de l'Incarnation en citant les paroles de $\mathrm{M}^{\mathrm{gr}}$ de Laval: « Nous tenons à bénédiction la connaissance qu'il a plu à Dieu de nous donner de la Mère Marie de l'Incarnation, première supérieure des Ursulines de Québec, l'ayant soumise à notre conduite pastorale: le témoignage que nous pouvons en rendre, est qu'elle était ornée de toutes les vertus dans un degré très éminent, surtout d'un don d'oraison si élevé, et d'une union si parfaite avec Dieu, qu'elle conservait sa présence au milieu de l'embarras des affaires les plus difficiles et les plus distrayantes comme parmi les autres occupations où sa vocation l'engageait. Parfaitement morte à elle-même, Jésus seul vivait et agissait en elle.

« Dieu l'ayant choisie pour l'établissement de l'Ordre de SainteUrsule au Canada, il l'a douée de la plénitude de l'esprit de cet Institut. C'était une supérieure parfaite, une excellente maîtresse de novices, elle était très capable de remplir tous les emplois d'une Communauté religieuse, sa vie commune à l'extérieur, était à l'intérieur toute divine, de sorte qu'elle était une règle vivante pour toutes ses sœurs. Son zèle pour le salut des âmes et particulièrement pour celui des sauvages, était si ardent qu'il semblait qu'elle les portât tous dans son cœur. Nous ne doutons pas que ses prières n'aient obtenu en grande partie les faveurs dont jouit maintenant l'Eglise naissante du Canada.» 


\section{III — LES HOSPITALIÈRES DE MONTRÉAL.}

\section{Introduction}

Il nous tarde de faire une visite à Montréal. La fondation de la Congrégation de Notre-Dame et des Hospitalières de Saint-Joseph de La Flèche remonte à l'année même de l'arrivée de $M^{\mathrm{gr}}$ de Laval, à 1659 . Les deux fondatrices, Jeanne Mance et Marguerite Bourgeoys, vivaient déjà au Canada; la première depuis 1642, venue avec M. de Maisonneuve, pour s'y dévouer aux soins des malades; la seconde depuis 1653, pour se consacrer à l'éducation de la jeunesse.

L'histoire de ces Communautés durant l'Episcopat de $\mathbf{M}^{\mathrm{gr}}$ de Laval a déjà été comme esquissée au du moins amorçée, dans la première partie de notre travail.

Une idée prédomine dans la pensée des autorités ecclésiastiques du temps: n'avoir à Québec et à Montréal qu'une seule et même Communauté enseignante; qu'une seule et même Communauté hospitalière.

Pour bien comprendre cette époque et la marche des événements, il faut d'une part se souvenir ou se rappeler la situation des deux postes stratégiques de Québec et de Montréal; les difficultés dans lesquelles on se trouvait, pour assurer les ressources et les revenus; et aussi songer à ce qu'apportent de nouveauté les deux Instituts en formation. Il ne conviendrait pas de porter un jugement trop catégorique sur les faits connus de cette histoire, on doit tenir aussi compte de la distance qui sépare la Nouvelle-France de l'Europe, et surtout la lenteur des communications. Nous sommes en 1659 , il y a trois cents ans.

Les difficultés en France et au Canada, l'établissement à Ville-Marie, l'approbation de l'Institut seront les principaux points évoqués dans cette partie.

\section{1) Les premières difficultés en France}

$\mathbf{M}^{\mathrm{gr}}$ de Laval était sur le point de s'embarquer pour la NouvelleFrance, et la Compagnie des Associés de Montréal concertait les moyens d'attirer les Hospitalières de Saint-Joseph de La Flèche à l'Hôtel-Dieu de Ville-Marie; une entente avait été conclue avec elles le 31 mars 1656, c'est-à-dire avant la nomination de Monsieur de Laval comme Vicaire apostolique pour le Canada.

Pendant son séjour à Québec, croyant que les Hospitalières de La Flèche ne pourraient subsister à l'Hôtel-Dieu de Ville-Marie, M. de Queylus aurait concerté avec les Pères Jésuites, le dessein d'en donner la conduite aux Hospitalières de Québec.

Cette crainte était sans fondement depuis que Madame la duchesse d'Aiguillon avait refusé de fonder les Hospitalières de Québec à VilleMarie, et que Madame de Bullion venait de donner une fondation pour y établir celles de La Flèche.

M. de Queylus était-il informé que Mlle Mance avait obtenu cette fondation? On écrivit au Canada à $\mathbf{M}^{\mathrm{gr}}$ de Laval de dissuader les 
Hospitalières de La Flèche de partir; entre autres motifs on insistait sur le fait que $M$. de Queylus avait appelé à Ville-Marie celles de Québec l'année précédente, en vue de les y établir dès que la Compagnie des Associés de Montréal consentirait à les recevoir.

$\mathrm{M}^{\mathrm{gr}}$ de Laval était donc déjà alerté et renseigné lorsque les Associés de Montréal le prièrent d'assister à leurs assemblées, afin de lui faire connaître le dessein qu'ils avaient eu jusqu'alors dans l'œuvre de Montréal, et ce qu'ils se proposaient de faire pour l'avenir. Il voulut bien assister à deux de leurs réunions; et dans l'une et dans l'autre, on fit part de la résolution que l'on avait prise d'envoyer cette même année les Hospitalières de La Flèche à Ville-Marie, mais toutes les fois qu'ils lui en parlèrent, sans les exclure positivement, le Prélat demandait qu'on différât leur départ jusqu'à l'année suivante, alléguant pour motif de ce délai la crainte de blesser M. de Queylus, qu'il croyait avoir d'autres desseins.

Aussi, les Associés de Montréal, convaincus que M. de Queylus entrerait volontiers dans les vues de M. de Bretonvilliers, son supérieur, et dans celle de la Compagnie, assurèrent toujours $\mathrm{M}^{\mathrm{gr}}$ de Laval, que leur confrère n'aurait pas d'autre sentiment que le leur, quand il en serait informé; et surtout lorsqu'il apprendrait que la Compagnie, à qui seule appartenait le droit de choisir des Hospitalières, avait passé un contrat en faveur de La Flèche.

Mais quelques raisons qu'ils pussent alléguer, $\mathrm{M}^{\mathrm{gr}}$ de Laval persistait toujours à demander que leur départ fut différé. Ces instances leur faisaient enfin soupçonner qu'il ne voulût, à la faveur de ce délai, ménager les moyens d'attirer à Ville-Marie les Hospitalières de Québec.

Voyant que celles de La Flèche avaient une fondation assurée, qui ne pouvait servir que pour elles seules, d'après les clauses mêmes du contrat, et que l'Hôtel-Dieu de Ville-Marie ne pouvait se passer plus longtemps d'Hospitalières, la Compagnie de Montréal pensait qu'il était de son devoir de s'opposer au projet du Prélat, en effectuant sans délai leur départ.

Des personnes chargées en France des affaires de $\mathrm{M}^{\mathrm{gr}}$ de Laval, leur auraient déclaré, lorsqu'elles étaient à La Rochelle, que si elles s'embarquaient pour Ville-Marie, on les obligerait de repasser la mer dans le courant de la même année.

\section{2) Les premières difficultés au Canada}

Aussitôt après leur débarquement à Québec, les Hospitalières de La Flèche se rendent à l'église paroissiale pour renouveler à NotreSeigneur, le sacrifice de leur vie. De là, elles vont offrir leurs hommages à $\mathbf{M}^{\mathrm{gr}}$ de Laval, qui les avait devancées à Québec depuis environ trois mois, et lui présentent une requête pour solliciter son approbation.

Sans répondre d'abord à leur requête, $\mathrm{M}^{\mathrm{gr}}$ de Laval les reçoit avec bonté, les congratule de leur courage, leur dit des paroles très obligeantes; les engage à visiter d'abord M. d'Argenson, gouverneur du Canada, puis les Religieuses Hospitalières de Québec et leur ordonne d'aller pren- 
dre leur logement chez les Religieuses Ursulines qui s'étaient empressées de demander cette faveur. M. Guillaume Vignal, l'ami du Monastère, arrivant de France avec les trois fondatrices de l'Hôtel-Dieu de Montréal, vient les présenter aux Religieuses. "Ces dignes Hospitalières, dit le Récit, passèrent ici plus d'un mois et elles nous édifièrent fort par leur vertu. N'étant pas encore voilées, elles portaient sur leur habit une petite guimpe carrée pour se distinguer des personnes séculières.»

Mais ces filles s'aperçoivent bientôt que toutes ces démonstrations de bienveillance avaient pour fin de les détacher de leur Institut, pour les incorporer à la Communauté des Hospitalières de Québec.

En arrivant à Québec, après une traversée orageuse, elles s'attendaient à rencontrer les plus fortes oppositions; elles ne furent pas trompées dans cette attente, dit Faillon.

La Sœur Morin, dans ses Annales, parle ainsi des instances qu'on leur fit dès leur arrivée. "Elles furent beaucoup pressées par $\mathrm{M}^{\mathrm{gr}}$ l'Evêque de Pétrée et par les Pères Jésuites, qui jugeant plus utile au bien du pays de n'avoir que les Hospitalières du même Institut, voulaient installer à Ville-Marie, celles de Québec, et obliger celles de La Flèche de quitter leur Institut, afin de s'unir aux Hospitalières de Québec, ou de retourner en France. Ils firent tout leur possible pour les engager à prendre l'un de ces deux partis, pensant rendre gloire à Dieu. Mais elles n'auraient pu embrasser l'Institut des Hospitalières de Québec, sans violer la protestation solennelle qu'elles avaient faite et signée avant le départ de La Flèche, de regarder jusqu'à la fin de leur vie, la Communauté de ce lieu, comme leur Maison-Mère, et d'observer invariablement les Constitutions de l'Institut de Saint-Joseph. Enfin, après tout ce qui avait eu lieu avant leur départ de France, elles ne pouvaient sagement se déterminer à y repasser, à moins que $\mathbf{M}^{\text {gr }}$ de Laval ne les y contraignît.»

La Sœur Morin assure que « $\mathrm{M}^{\mathrm{gr}}$ de Laval, grand serviteur de Dieu et homme tout apostolique, ne fit jamais violence au sentiment des Hospitalières de La Flèche, se contentant de leur dire qu'elles lui feraient un grand plaisir de faire ce que M. de Queylus souhaitait, qu'il voyait aussi leurs avantages, qu'elles attireraient ainsi sur elles et sur leurs entreprises, la protection de cet homme, qui était en pouvoir de leur faire beaucoup de bien, si elles voulaient entrer dans ses sentiments; qu'il lui avait donné parole de ne les point établir dans les formes et qu'il ne le ferait pas; mais qu'elles lui feraient grand plaisir en s'agrégeant à l'autre Institut.»

$M^{\mathrm{gr}}$ de Laval espérait toujours qu'elles finiraient par consentir à s'affilier à celles de Québec, de manière à ne former qu'un seul Institut; il le désirait d'autant plus qu'il se voyait dans la nécessité de rappeler les deux Religieuses de Québec, qui se trouvaient à l'Hôtel-Dieu de VilleMarie depuis l'année précédente, n'ayant pas de quoi les y faire subsister.

$\mathrm{M}^{\mathrm{gr}}$ de Laval ne pouvait goûter leurs Constitutions, rédigées par un homme marié, M. de la Dauversière, et il voyait dans les observances qu'elles prescrivaient des choses si extraordinaires et si inusitées, qu'il douta s'il pourrait jamais les approuver. Dans la pensée de leur fonda- 
teur, ces Hospitalières avaient été instituées pour être un jour de vraies Religieuses, et celles qui arrivaient de France se considéraient déjà comme telles, en attendant l'approbation du Souverain Pontife. $\mathbf{M}^{\mathrm{gr}} \mathrm{de}$ Laval leur objectait que leurs Constitutions ne supposaient que les vœux simples et que d'ailleurs leur costume n'était pas distinct de celui des personnes séculières, ce qui était vrai alors. Son intention, continue Faillon, était que toutes les Hospitalières qui seraient en Canada fussent vêtues de la même sorte et suivissent les mêmes Règles; comme il y avait déjà à Québec des Hospitalières venues de Dieppe, il fit tous ses efforts pour que celles de La Flèche prissent le costume des autres et embrassent le même Institut.

Mais loin de se laisser abattre par tous les assauts qu'elles ont à soutenir, elles n'en deviennent que plus résolues à sacrifier à Dieu leur repos, leur santé et leur vie. C'est qu'elles considèrent toutes ces oppositions comme autant d'épreuves par où Dieu veut les faire passer pour purifier leur dévouement et les rendre dignes de servir d'instruments à l'accomplissement de son œuvre.

Cette conduite de Dieu sur elles explique comment $\mathbf{M}^{\mathrm{gr}}$ de Laval, ce Prélat si pieux, et comment les Pères Jésuites, si zélés pour le bien de la religion, pouvaient se montrer cependant opposés à leur établissement à Ville-Marie.

\section{3) L'établissement à Ville-Marie.}

Il est vrai qu'elles étaient parties de France quoique $\mathrm{M}^{\mathrm{gr}}$ de Laval eût demandé à la Compagnie des Associés de Montréal de différer leur départ jusqu'à l'année suivante.

$\mathrm{M}^{\mathrm{gr}}$ de Laval, qui ne voulait en tout que le bien de la religion, après un mois d'incertitude sur leur avenir, finit par renoncer à son projet d'union, et donne aux Hospitalières de La Flèche, le 2 octobre 1659, de magnifiques lettres d'obédience pour aller prendre la direction de l'Hôtel-Dieu de Montréal. Ce document est rempli de sentiments bien paternels. On aurait fait de nouveaux efforts pour les obliger à repasser en France, elles l'auraient fait sans la fermeté de Mère de Brésoles, qui refuse constamment, et si $\mathrm{M}^{\mathrm{gr}}$ de Laval ne leur eût été favorable pour dissiper l'orage "de quoi, dit Faillon, Montréal lui fut bien obligé, parce qu'il contribua ainsi à lui donner des bonnes Filles ». Les noms de ces héroïnes, dit l'abbé Auguste Gosselin, méritent de passer à la postérité: les Mères Judith Moreau de Brésoles, Catherine Macé, Marie Maillet.

Une grande épreuve vient assaillir l'Hôtel-Dieu de Montréal à ses débuts. Monsieur de la Dauversière, fondateur des Hospitalières de La Flèche, meurt au début de novembre 1659, laissant dans ses affaires un déficit considérable; il faisait perdre à l'Hôtel-Dieu les 20,000 livres de la fondation faite par Madame de Bullion; la fondation ayant été confondue dans la succession, fut saisie.

Les Hospitalières de Montréal n'ayant plus rien pour subsister, les Associés de Montréal leur écrivent de repasser en France. Elles y seraient repassées, mais les Messieurs de Saint-Sulpice et les citoyens de Montréal, qui leur étaient tous dévoués, adressèrent à l'Evêque de Québec 
de vives instances, auxquelles Monseigneur voulut bien avoir égard. Ainsi, elles résolurent de rester quand même au Canada, et de s'appuyer uniquement sur les secours de la Providence.

$\mathbf{M}^{\mathrm{gr}}$ de Laval les encourageait dans leurs résolutions: "Les bonnes Mères Hospitalières, écrit Mère Marie de l'Incarnation, qui vinrent l'année dernière s'établir à Montréal, ont été à la veille de repasser en France, leur fondation étant saisie et regardée comme perdue, mais $\mathbf{M}^{\mathrm{gr}}$ notre Prélat les a retenues sur la requête qui lui a été présentée par les habitants de Montréal, car ce sont des Filles d'une grande vertu et d'édification.»

\section{4) L'approbation de l'Institut}

Qui n'admirerait ici la prudence de $\mathrm{M}^{\mathrm{gr}}$ de Laval ? Avant d'autoriser définitivement une nouvelle Communauté dans son diocèse, il veut s'assurer que c'est bien dans les vues de la Providence. Il reconnaît toutefois indirectement les Hospitalières de Montréal lorsque la permission est donnée à M. Gabriel Souart de recevoir les vœux de la Sœur Morin; et d'une manière plus éclatante, le 17 octobre 1671, en lui accordant cette fois tous les pouvoirs nécessaires pour admettre les Religieuses à la profession des vœux solennels, qu'elles devaient faire depuis que la Communauté de La Flèche avait été érigée en Ordre Religieux par le Souverain Pontife. "Par là, dit la Sœur Morin, le Prélat acheva cet établissement pour ce qui est du spirituel de manière à ne pouvoir s'en dédire.»

$\mathrm{M}^{\mathrm{gr}}$ de Laval montre aussi en plusieurs rencontres l'intérêt qu'il porte à leurs affaires temporelles. On trouve dans les Archives du Séminaire de Québec, un grand nombre de documents qui prouvent le soin avec lequel Monseigneur s'occupait de ses Communautés.

Voyant la grande pauvreté où elles se trouvaient réduites, $\mathrm{M}^{\mathrm{gr}} \mathrm{de}$ Laval songe à leur faire rendre par le Séminaire de Saint-Sulpice une somme de 22,000 livres que Madame de Bullion avait donnée pour l'Hôtel-Dieu, et que Mlle Mance avait passée de bonne foi, mais sans autorisation écrite aux Associés de Montréal, pour la défense de l'Ile contre les Iroquois en 1653.

Le 6 novembre 1678, $\mathrm{M}^{\mathrm{gr}}$ de Laval leur donne une lettre les exemptant de payer la dîme sur toutes les propriétés qu'elles possédaient «en considération des grands services qu'elles avaient rendus au pays ».

En 1695, retiré depuis longtemps des affaires et vivant humblement dans son Séminaire de Québec, il apprend qu'un incendie terrible vient de détruire de fond en combles l'Hôtel-Dieu de Montréal. Le vieil Evêque s'est dépouillé de tout, il a cédé tous ses biens au Séminaire, et n'a plus à sa disposition qu'un petit revenu annuel bien modique, dont il emploie la plus grande partie en bonnes œuvres; il trouve moyen de prouver à ces bonnes Sœurs qu'il compatit à leur détresse et leur fait parvenir la somme de 200 livres; témoignage non équivoque de l'affection que le saint Prélat portait aux Communautés de Montréal.

Leur existence civile est reconnue en 1669. L'intendant Talon ayant fait convoquer une assemblée des citoyens de Montréal, il est unanimement 
convenu de demander au Roi des Lettres patentes pour l'Hôtel-Dieu. $\mathbf{M}^{\mathbf{g r}}$ de Laval appuie la demande des citoyens. M. Gabriel Souart passe en France en 1667, présente la requête, et obtient par l'entremise du ministre Colbert, les Lettres patentes demandées.

\section{5) La fondatrice Jeanne Mance}

Dans les Lettres, non plus que dans la requête des citoyens, le nom de Mlle Mance, la véritable fondatrice de l'Hôtel-Dieu de Ville-Marie, n'est mentionné. Cette humble et sainte fille s'était complètement effacée, depuis qu'elle avait réussi à confier à des Religieuses la fondation de cette maison. Elle continue cependant de demeurer avec elles, gardant, d'après les termes du contrat de fondation de l'Hôtel-Dieu, le titre de fondatrice et conservant l'administration jusqu'à sa mort, arrivée en juin 1673 .

Il est à regretter qu'on ne nous ait conservé aucun détail sur ses dernières années ni sur les circonstances de sa sainte mort. Tout ce que nous savons, c'est que Dieu acheva de la sanctifier par de longues et continuelles maladies; que cette fille admirable édifia toute la Colonie par ses grandes vertus et qu'elle mourut en odeur de sainteté; c'est le témoignage que rend à sa mémoire la Mère Juchereau de Saint-Ignace, dans les Annales de l'Hôtel-Dieu de Québec.

"Son cœur délicat lui inspire l'heureuse pensée de nommer $\mathrm{M}^{\mathrm{gr}}$ de Laval son exécuteur testamentaire, geste émouvant qui marque son humble déférence envers le chef religieux de la Colonie.»

\section{IV. - $\mathrm{M}^{\mathrm{gr}}$ DE LAVAL ET LA CONGRÉGATION DE NOTRE-DAME.}

Un auteur affirme que $\mathrm{M}^{\mathrm{gr}}$ de Laval aurait béni dans l'église de Saint-Germain-des-Prés à Paris, quatre jeunes filles. C'était aussitôt après son sacre, avant de venir à Québec prendre possession de son siège. Dès ce moment elles se considéraient comme les filles aînées du premier Evêque de l'Amérique du Nord; l'une s'appelle Marguerite Bourgeoys - Belle et touchante évocation!

Un simple rappel de l'esprit du temps et des difficultés dans les débuts de la Congrégation de Notre-Dame, la première approbation et l'épreuve de 1683, feront l'objet de cette dernière partie de notre travail.

\section{INTRODUCTION}

Le désir de voir régner partout dans son diocèse le même esprit et la même direction, faisait croire à $\mathrm{M}^{\mathrm{gr}}$ de Laval qu'il aurait été avantageux que l'éducation de la jeunesse fut confiée à Montréal et à Québec, à la même Communauté.

C'est ainsi qu' " entre la première installation en octobre et la fin de l'année 1659 » les Filles de Marguerite Bourgeoys durent faire face à une difficulté qui mettait en péril leur existence même. Les assimiler aux Ursulines et n'avoir dès lors qu'un seul Institut chargé de toute l'instruction des filles dans la Colonie, cette idée nourrie par $\mathrm{M}^{\mathrm{gr}}$ de Laval, sera aussi celle de son successeur, $\mathbf{M}^{\text {gr }}$ de Saint-Vallier. "On 
nous presse de nous établir à Montréal, écrivait Mère Marie de l'Incarnation, mais nous ne sommes pas en état de le faire. On ne peut rien faire ici sans le secours du temporel.»

«Ce qui maintenant et plus tard, allait protéger les Institutrices de Ville-Marie toutes les fois qu'on voudrait les supprimer, c'était leur double condition de filles pauvres et séculières.

«Alors qu'un établissement de Religieuses Ursulines ne pouvait se faire à Montréal, sans dotation ni cloître, les Filles de Marguerite Bourgeoys parce que pauvres, ne grèveraient jamais le budget de qui que ce soit; et parce que non cloîtrées, elles sauraient aller partout où l'exigeraient les besoins d'une Colonie mouvante. Souplesse et adaptation à la nature du temps, telles étaient donc les deux prérogatives dont la fondatrice, avec son sens pratique, allait marquer son Institut naissant pour en assurer par là la survie et même la longévité."

La simple permission donnée à Mère Bourgeoys d'instruire les enfants dans l'étendue $d u$ diocèse, pouvait-elle faire entrevoir quelque dessein d'établir les Ursulines à Ville-Marie ? ou peut-être eût-elle pour motif l'incertitude où $\mathbf{M}^{\mathrm{gr}}$ de Laval était encore sur l'état futur de la Congrégation, jugeant qu'il n'était pas prudent de l'ériger en Communauté avant qu'elle ne donne des garanties de son avenir ?

\section{1) Les débuts}

Dès 1660 , lors de sa première visite à Montréal, $\mathrm{M}^{\mathrm{gr}}$ de Laval trouve Mère Bourgeoys et les trois compagnes amenées de France en 1659, occupées à instruire les jeunes filles de cette ville, et à former sa Communauté. Il ne veut pas croire tout d'abord au succès de leur entreprise. Il les bénit cependant et les laisse continuer leur œuvre bienfaisante. Dans la "Relation de 1660 ", envoyée à Rome, il parle avec éloge de leurs travaux: "les maîtresses au nombre de quatre, qui se consacrent à Montréal à l'éducation des jeunes personnes, ne sont pas religieuses, ne font point de vœu, au moins en public. Elles vivent cependant avec piété, et sont d'une grande édification.»

La «Relation sur l'Etat de l'Eglise du Canada» en 1661 mentionne des personnes en mission à Trois-Rivières: "Nous avons envoyés récemment dans ce lieu, dit l'Evêque, de jeunes personnes comme maîtresses pour prendre soin des petites filles, afin qu'elles leur enseignent tout ce qui est nécessaire aux chrétiens de savoir; en attendant que le temps et l'occasion favorables se présentent pour établir dans ce lieu, des Religieuses de Sainte-Ursule.»

Si ces maîtresses étaient des Sœurs de la Congrégation, comme il est naturel de le penser, les dernières paroles pourraient montrer peut-être le dessein que $\mathbf{M}^{\text {gr }}$ de Laval avait déjà conçu de les faire entrer dans l'Institut des Ursulines, au lieu de leur permettre d'en former un à part; ce qui fut un grand sujet d'épreuves pour la Sœur Bourgeoys, qui sentait toujours que ce Prélat désirait user à leur égard comme envers les Hospitalières de Ville-Marie; les unir à celles de Québec, afin que de la sorte il n'y eût qu'un seul et même Institut. 


\section{2) La première approbation}

Neuf ans plus tard, lors de la cinquième visite à Montréal en 1669, $\mathrm{M}^{\mathrm{gr}}$ de Laval trouve l'institution si solidement établie qu'il n'hésite pas à lui donner son approbation. Il visite avec intérêt les nouvelles constructions que la Sour a fait bâtir, et il est tellement satisfait du succès de la Congrégation pour l'instruction des jeunes filles, qu'il lui permet de prendre son essor dans toutes les parties de son diocèse.

« Le 20 mai 1669, écrira-t-on, le chef de l'Eglise en Nouvelle-France donne un mandat d'institutrices aux Filles de Marguerite Bourgeoys pour la Colonie entière. Un geste de cette nature constituait déjà une certaine approbation de l'œuvre accomplie; de plus, il était fait en faveur d'une équipe de femmes menant depuis dix ans la vie de religieuses en Communauté. De ce mandat sortira l'érection canonique de 1676.»

$\mathrm{M}^{\mathrm{gr}}$ de Laval, récemment nommé évêque de Québec, revient prendre contact avec la Mère Bourgeoys et ses Filles en 1676. Il se rend compte des progrès réalisés et rédige un mandement érigeant la Congrégation en "une maison religieuse canoniquement existante dans l'Eglise ".

La Communauté naissante étant approuvée par $\mathrm{M}^{\mathrm{gr}}$ de Laval, Marguerite Bourgeoys se rend en France l'année suivante, pour faire reconnaître civilement l'existence de son Institut; elle obtient du Roi l'objet de sa demande dès le mois de mai 1671. Les Lettres patentes, accordées en termes des plus flatteurs, approuvant l'établissement des Sœurs de la Congrégation, furent enregistrées d'abord au Parlement de Paris, puis au Conseil Souverain de Québec et à Montréal.

Monseigneur invite Marguerite Bourgeoys à fonder à Québec, sa ville épiscopale, une maison de son Ordre. Elle quitte Montréal au plus fort de l'hiver, " fait à pied, dit Latour, plus de soixante lieues - cent quatre-vingts milles sur les glaces et dans la neige. - Elle s'y donne des mouvements infinis, portant elle-même de la Haute-ville à la Basse où sa maison était située, les meubles qu'on lui donnait; et elle passa la nuit entière du jeudi au vendredi saint à genoux et immobile devant le Saint-Sacrement, malgré l'extrême rigueur du froid.»

Les colons de Ville-Marie font une tentative en 1670 pour amener les Ursulines dans leurs murs. Le Montréal de 1670 semblait avoir besoin d'un pensionnat qui pût donner aux filles de condition sociale moyenne, une formation plus achevée que ne le pouvait faire l'humble école de la commune; cette amélioration paraissait exigée par l'accroissement de la population dans le petit bourg et par l'apparition d'une classe sociale de condition plus aisée.

"En principe, continue l'historien de Mère Bourgeoys, les Ursulines ne répugnaient pas au projet, bien que dans les circonstances, elles le trouvassent prématuré, et c'est probablement ce qui empêcha d'y donner suite.

"La venue dans le petit Montréal, d'un contingent de Religieuses authentiques et jouissant d'une réputation d'éducatrices bien établie dans le pays, ne pouvait que porter préjudice aux Congrégations de Ville- 
Marie. Cela, Marguerite Bourgeoys ne pouvait l'ignorer, elle qui avec Marie Raisin, Anne Hioux et Catherine Crolo, ne constituait après tout, qu'une misérable équipe de quatre humbles filles du monde s'improvisant institutrices.»

\section{2) L'épreuve de 1683}

L'incendie de la maison de Mère Bourgeoys, par une nuit de décembre 1683, allait réduire les Sœurs au dénuement le plus entier. "Le désastre était complet, non seulement la maison, les meubles, tout devint la proie des flammes, deux Religieuses ne purent s'enfuir à temps et furent retrouvées carbonisées.»

Le pieux Prélat accourut et devant ce qui semblait devoir être la ruine totale de l'œuvre, croyant voir dans ce dénuement une indication providentielle, - leur suggère encore une fois une fusion avec les Ursulines de Québec Cette proposition afflige beaucoup la Mère Bourgeoys.

Il revenait pour ainsi dire instinctivement à la première idée qu'il avait toujours caressée: n'avoir qu'une seule Communauté de femmes enseignantes dans son diocèse. Il oubliait une fois de plus que les Religieuses Ursulines étaient cloîtrées et ne seraient peut-être pas aussi utiles que celles de la Congrégation pour l'éducation des enfants de la campagne.

Bien que Mère Bourgeoys fut très soumise à ses supérieurs et que même, - au témoignage de $M$. de Maizerets, grand vicaire de $\mathbf{M}^{\mathrm{gr}}$ de Laval, - elle excellait en obéissance envers eux, elle croit néanmoins être obligée dans cette circonstance, avec beaucoup de respect, mais avec énergie, de représenter à ce Prélat, que le bien qu'elle se propose de faire avec ses filles, n'est pas compatible avec les Règles d'un autre Institut et notamment avec celles d'une Communauté cloîtrée. Que ce serait détruire entièrement les vues qu'elle croyait lui avoir été inspirées de Dieu.

Non seulement elle veut honorer la sainte Vierge par la fondation de son Institut, mais encore rendre la vie religieuse accessible à une foule de jeunes personnes, qui, trop délicates pour affronter les austérités du cloître, ou trop peu favorisées de la fortune pour fournir une dot, seraient à jamais privées du bonheur de se consacrer à Dieu.

Elle ajoutait qu'elle faisait si peu cas des richesses qu'elle irait prendre sur ses épaules une fille qui, n'ayant pas de quoi se vêtir, aurait d'ailleurs une bonne volonté et une vraie vocation. $\mathrm{M}^{\mathrm{gr}}$ de Laval était trop homme de Dieu pour ne pas comprendre pareille pensée.

L'Evêque de Québec « qui avait toujours une très grande estime pour la vertu de cette bonne sour, dit Faillon, et qui songeait alors à se démettre de ses fonctions, en demandant au Roi un coadjuteur, ne crut pas devoir insister, et abandonna l'avenir de la Congrégation à la Divine Providence ".

C'était l'abandonner entre bonnes mains. La Congrégation se releva bientôt de ses ruines, et se rétablit, non pas au même endroit, mais 
un peu plus haut à côté de l'Hôtel-Dieu, sur des bases plus larges que celles qu'elle avait auparavant.

Lorsque $\mathrm{M}^{\mathrm{gr}}$ de Saint-Vallier vient pour la première fois au Canada deux ans après l'incendie (1685), la Mère Bourgeoys est alors entourée de quarante compagnes; et le Prélat est frappé de la facilité avec laquelle cette maison s'est relevée du désastre de l'incendie. "C'est une merveille, écrit-il, dans sa Relation: «Etat présent de l'Eglise et de la Colonie française dans la Nouvelle-France», c'est une merveille qu'elles (les Sœurs de la Congrégation) aient pu subsister après l'accident qui leur arriva les 6-7 décembre 1683, toute leur maison fut brûlée en une nuit; elles ne sauvèrent ni leurs meubles, ni leurs habits, trop heureuses de se sauver elles-mêmes, encore y en eut-il deux d'entre elles qui furent enveloppées dans les flammes. Le courage de celles qui échappèrent les soutint dans leur extrême pauvreté; et quoiqu'elles fussent plus de trente, la divine Providence pourvut à leurs pressantes nécessités. Il semble même que cette calamité n'ait servi qu'à les rendre plus vertueuses et plus utiles au prochain, a car il n'y a point de bien qu'elles n'aient entrepris depuis ce temps-là 》. "Mère Bourgeoys était une personne capable en toutes choses. Les affaires temporelles et spirituelles réussissaient toujours bien entre ses mains, parce que c'est l'amour du Seigneur qui la faisait agir et qui lui donnait l'intelligence. On aurait peine à trouver une fille comme celle-ci, qui a tout le caractère de la femme forte de l'Evangile.»

\section{4) La fondatrice Marguerite Bourgeoys}

Marguerite Bourgeoys appartient à l'histoire de Ville-Marie, dira le Chanoine Groulx, à cette histoire unique qui commence un jour de février 1641, par une messe de Monsieur Olier, à l'autel de la Sainte Vierge, en la Cathédrale de Paris. Là, dans ce décor choisi par eux, s'étaient donné rendez-vous, auprès du fondateur de Saint-Sulpice, quelques Français de foi magnifique qui voulaient cette chose: bâtir dans le Nouveau-Monde une cité à Marie. Marguerite Bourgeoys fut bientôt de ce grand dessein, "et son ouvre fut entreprise de haute éducation populaire et de belle vaillance surnaturelle ».

$\mathrm{M}^{\mathrm{gr}}$ de Laval portera le jugement suivant. - « Mère Bourgeoys était simple et humble. Dieu lui a fait bien des grâces, elle sera auprès du Seigneur, une puissante protectrice de votre maison.»

$\mathrm{M}^{\mathrm{gr}}$ de Saint-Vallier a loue sa foi vive et son ardente charité pour Dieu et pour le prochain, surtout la vie cachée et recueillie qu'elle avait menée depuis sa démission et ce qui lui faisait plus d'impression que toutes ses autres vertus ». M. de Maizerets, supérieur du Séminaire de Québec, l'un des fils spirituels les plus respectés de $\mathbf{M}^{\mathrm{gr}}$ de Laval, traçait ces lignes qui pourraient être placées en épigraphe sur toutes les vies de la Vénérable Mère: " Elle était remplie de l'esprit de Dieu, elle excellait surtout en humilité, douceur, obéissance à ses supérieurs et un grand abandon à la Divine Providence qui lui donnait un cœur généreux, capable de toutes les entreprises.» 
Depuis, l'Eglise a parlé. - Le 12 novembre 1950, Rome proclame "Bienheureuse " Marguerite Bourgeoys, fondatrice de la Congrégation de Notre-Dame au Canada.

Notre conclusion à ce long travail est empruntée à deux historiens canadiens: "Nos fondateurs nous apparaissent grands, non seulement par le labeur qu'ils mettent dans leur œuvre, labeur effrayant, mais aussi et peut-être plus, par les lointaines visées qu'ils y renferment. Parce qu'ils travaillent pour un long avenir, tous les jours ils apprennent à se dépasser, ces hommes et ces femmes d'autrefois savent bel et bien qu'ils fondent un pays et une race. De ce haut devoir, ils acceptent pleinement les conséquences; la race qu'ils fondent, ils la veulent noble, loyale et pure, à la mesure de ses origines.»

« Dieu n'a jamais été, envers notre pays, avare de saintes et nobles âmes. A côté de nos hardis défricheurs, moins laboureurs encore que soldats, de nos hommes de guerre intrépides, de nos missionnaires, de faibles femmes se sont rencontrées qui ne leur ont cédé ni en vaillance, ni en générosité, ni en héroïsme, ni en sainteté. Et ce n'est pas une des moindres gloires de $\mathbf{M}^{\text {gr }}$ de Laval d'avoir tenu sous sa houlette pastorale une Marie de l'Incarnation, une Catherine de Saint-Augustin, une Jeanne Mance, une Marguerite Bourgeoys.»

Léo Hudon, s.j.

\section{Bibliographie}

(Liste des ouvrages consultés)

Annales de l'Hôtel-Dieu de Québec (Les), 1636-1716.

Composées par les Révérendes Mères Jeanne-Françoise Juchereau de SaintIgnace et Marie-Andrée Duplessis de Sainte-Hélène, anciennes religieuses de ce Monastère. Editées dans le Texte original avec une Introduction et des Notes par Dom Albert Jamet, de l'Abbaye de Solesmes. A l'Hôtel-Dieu de Québec MCMXXXIX.

BarabéE, Père Paul-Henri, o.m.i. - Quelques figures de notre Histoire. Ottawa, Editions de l'Université, 1941.

Cascrain, l'abbé Henri-Raymond. - Histoire de l'Hôtel-Dieu de Québec. Québec, Léger Brousseau, 1878.

Cascrain, l'abbé Henri-Raymond. - Histoire de la Mère Marie de l'Incarnation. Québec, Desbarats, 1864.

Chapot, l'abbé Léon. - Histoire de la Vénérable Mère Marie de l'Incarnation. Paris, Poussielgue, 1892, 2 vol.

Chiarlevoix, Père François-Xavier de, s.j. - Histoire et Description générale de la Nouvelle-France, etc., Paris, 1744, 6 vol.

Cḥarron, M. Yvon, p.s.s. - Mère Bourgeoys. Montréal, Beauchemin, 1950.

DANEMARIE, Jeanne. - L'infirmière exemplaire, Jeanne Mance au Canada, 1606-1673. Paris, Bonne Presse, 1938.

Danemarie, Jeanne. - Au Canada avec Marguerite Bourgeoys, Paris, Editions de l'Arc, 1950.

Daveluy, Marie-Claire. - Jeanne Mance, suivie d'un essai généalogique sur les Mance de Mance par M. Jacques Laurent, conservateur à la Bibliothèque de Dijon. Montréal, Albert Lévesque, 1934.

Farllon, Etienne-Michel, p.s.s. - Histoire de la Colonie française en Canada. Ville-Marie, 1865,3 vol. 
Fanlon, Etienne-Michel, p.s.s. - Vie de Mlle Mance et l'Histoire de l'Hôtel-Dieu de Ville-Marie dans l'Isle de Montréal, en Canada. Ville-Marie, 1854, 2 vol.

Farloon, Etienne-Michel, p.s.s. - Vie de la Sour Bourgeoys, fondatrice de la Congrégation de Notre-Dame de Ville-Marie, en Canada, suivie de l'Histoire de cet Institut jusqu'à ce jour. Ville-Marie, 1853, 2 vol.

Gosselrn, l'abbé Auguste. - Vie de $\mathbf{M}^{\mathrm{gr}}$ de Laval, premier évêque de Québec, 1622-1708. Québec, Demers, 1890, 2 vol.

Groulx, Chanoine Lionel. - Marguerite Bourgeoys, dans Notre Maître le Passé, pp. 31-41. Montréal, 1924.

Hudon, Père Léonidas, s.j. - La vie de la Mère Marie-Catherine de Saint-Augustin, 1632-1668. Paris, Editions Spes, 1925.

JAMET, Dom Albert, o.s.b. - Marie de l'Incarnation, Ursuline de Tours; fondatrice des Ursulines de la Nouvelle-France. Ecrits spirituels et historiques publiés par Dom Claude Martin de la Congrégation de Saint-Maur, réédités par Dom Albert Jamet de la Congrégation de France avec des annotations critiques des pièces documentaires et une biographie nouvelle. Paris, Desclée de Brouwer, 1929-1939, 4 vol.

JAMET, Dom Albert, o.s.b. - Marguerite Bourgeoys, 1620-1700. - Montréal, 1942,2 vol.

MARIE-EMMANUEL, Seur, o.s.u. - Marie de l'Incarnation d'après ses lettres. Ottawa, Editions de l'Université, 1946.

Mondoux, Sœur, r.h.s.j. - L'Hôtel-Dieu premier hôpital de Montréal, 1642 1763, préface de $\mathbf{M}^{\mathrm{gr}}$ Olivier Maurault, p.s.s., Montréal, MCMXLII.

Morin, Sour Marie, r.h.s.j. - Annales de l'Hôtel-Dieu de Montréal, collationnées et annotées par MM. Aegidius Fauteux, E.-Z. Massicotte, Camille Bertrand, avec une introduction par M. Victor Morin, dans les Mémoires de la Société Historique de Montréal, deuxième livraison, Montréal, 1921. (Le texte original est conservé aux Archives de l'Hôtel-Dieu de Montréal.)

Pouliot, Père Léon, s.j. - Etude sur les « Relations 》 des Jésuites de la NouvelleFrance, (1632-1672), Montréal, 1940.

Ragueneau, Père Paul, s.j. - La Vie de Mère Catherine de Saint-Augustin, Paris, Lambert, 1671, (seconde édition, 1923).

Richaudeau, l'abbé F. - Vie de la Révérende Mère Marie de l'Incarnation, Ursuline, (Marie Guyard). Tournai, Casterman, 1873.

Richaudeau, l'abbé F. - Lettres de la Révérende Mère Marie de l'Incarnation. Tournai, Casterman, 1876, 2 vol.

Rumilly, Robert. - Marguerite Bourgeoys. Paris, Editions Spes, 1936.

Ursulines de Québec (Les) depuis leur établissement jusqu'à nos jours. Québec, Darveau, 1878, 4 vol. 